\title{
The Stability Analyses of the Mathematical Models of Hepatitis C Virus Infection
}

\author{
Maureen Siew Fang Chong ${ }^{1}$, Masitah Shahrill ${ }^{1}$, Laurie Crossley ${ }^{2}$ \& Anotida Madzvamuse ${ }^{2}$ \\ ${ }^{1}$ Sultan Hassanal Bolkiah Institute of Education, Universiti Brunei Darussalam, Bandar Seri Begawan, Brunei \\ Darussalam \\ ${ }^{2}$ University of Sussex, School of Mathematical and Physical Sciences, Pevensey III, Brighton, BN1 9QH, UK \\ Correspondence: Masitah Shahrill, Sultan Hassanal Bolkiah Institute of Education, Universiti Brunei Darussalam, \\ Jalan Tungku Link, Gadong, BE 1410, Bandar Seri Begawan, Brunei Darussalam. Tel: 673-246-3001. Fax: \\ 673-246-1003. E-mail: masitah.shahrill@ubd.edu.bn
}

Received: October 26, 2014

Accepted: November 10, 2014 Online Published: January 19, 2015

doi:10.5539/mas.v9n3p250

URL: http://dx.doi.org/10.5539/mas.v9n3p250

\begin{abstract}
There are two mathematical models of Hepatitis C virus (HCV) being discussed; the original model of HCV viral dynamics (Neumann et al., 1998) and its extended model (Dahari et al., 2007). The key aspects of the mathematical models have provided resources for analysing the stability of the uninfected and the infected steady states, in evaluating the antiviral effectiveness of therapy and for estimating the ranges of values of the parameters for clinical treatment. The original model is considered to be a deterministic model because of the predictive nature of the antiviral therapy within the constant target cells. Numerical simulations are carried out in the extended model, to explain the stability of the steady states in the absence or existence of migration in hepatocytes and, drug efficacy in treating HCV infection.
\end{abstract}

Keywords: mathematical model, extended model, stability analysis, HCV

\section{Introduction}

Hepatitis $\mathrm{C}$ virus (HCV) infection is a major primary cause of chronic liver disease, liver cancer and liver cirrhosis. According to recent statistics, approximately 130 and 170 million people are chronically infected by HCV worldwide (WHO, 1999). The causative agent of HCV was only identified in 1989, with modern techniques of molecular cloning and, identifying the genome of the previously uncharacterised non-A and non-B hepatitis virus. The HCV are clustered into six genotypes with varying prevalence around the world (Table 1) and some genotypes showed evidence of being less responsive to interferon therapy (Di Bisceglie, 1998). Since most patients are infected with a single genotype of HCV, then phylogenetic analysis of the nucleotide sequence of $\mathrm{HCV}$ is highly feasible for diagnostic testing.

Table 1. HCV genotypes, their distribution and clinical significance (Source: Di Bisceglie, 1998)

\begin{tabular}{|c|c|c|}
\hline $\begin{array}{l}\text { Genotypes } \\
\text { of HCV }\end{array}$ & Geographical predominance & Clinical significance \\
\hline $1 \mathrm{a}$ & $\begin{array}{l}\text { USA and developed western } \\
\text { countries }\end{array}$ & Less responsive to interferon therapy \\
\hline $1 b$ & USA, Japan, Europe & $\begin{array}{l}\text { Less responsive to interferon therapy and more likely } \\
\text { to lead to cirrhosis or hepatocellular carcinoma }\end{array}$ \\
\hline 2 & $\begin{array}{l}\text { Most developed countries, but } \\
\text { not very common }\end{array}$ & Responsive to interferon therapy \\
\hline 3 & $\begin{array}{l}\text { Rising in prevalence among } \\
\text { injection-drug users }\end{array}$ & Responsive to interferon therapy \\
\hline 4 & $\begin{array}{c}\text { Confined to the middle east and } \\
\text { north Africa }\end{array}$ & Less responsive to interferon therapy \\
\hline 5 & South Africa & Responsive to interferon therapy \\
\hline 6 & Asia & Responsive to interferon therapy \\
\hline
\end{tabular}


The HCV is a positive-stranded RNA virus of the flaviviridae family which appears to have a narrow host range (WHO, 2014). Each single-stranded RNA genome of HCV is approximately 10,000 nucleotides in length that codes for both structural and non-structural proteins. The structural proteins are coated by two envelope proteins, $\mathrm{E} 1$ and E2 that contain a hypervariable portion of the E2/NS1 region. Within this region, the virus seemed to accumulate mutations easily under immune pressure and resulting in neutralisation and viral persistence (Di Bisceglie, 1998). While the non-structural proteins seemed to be significant for viral replication (Figure 1 and Figure 2). In clinical practice, the usual approach for testing HCV infection (Table 2) is to test initially for antibodies to $\mathrm{HCV}$ (anti-HCV), then to use HCV ribonucleic acid (RNA) to quantify the HCV RNA levels before providing and monitoring HCV treatment (Strader et al., 2004).

Table 2. The diagnostic tests in Hepatitis C. (Source: Di Bisceglie, 1998)

\begin{tabular}{lllll}
\hline Category & ELISA & RIBA & HCV RNA & ALT \\
\hline Chronic Hepatitis C & Positive & Positive & Positive & Raised \\
Hepatitis C carrier & Positive & Positive & Positive & Normal \\
Recovered HCV infection & Positive & Positive & Negative & Normal \\
False positive anti HCV & Positive & Negative & Negative & Normal \\
\hline
\end{tabular}

Note: ELISA: anti HCV by enzyme linked immunoassay; RIBA: anti HCV by recombinant immonoblot assay (identifies antibodies which react with individual HCV antigen); and ALT: alanine aminotransferase (commonly measured clinically as part of a diagnostic test to determine liver health).

Viral dynamics is rapid for HCV with production of $10^{12}$ virions daily (Table 3 ) but the free viral particles have a short half-life, between 2 and 3 hours (Neumann et al., 1998).

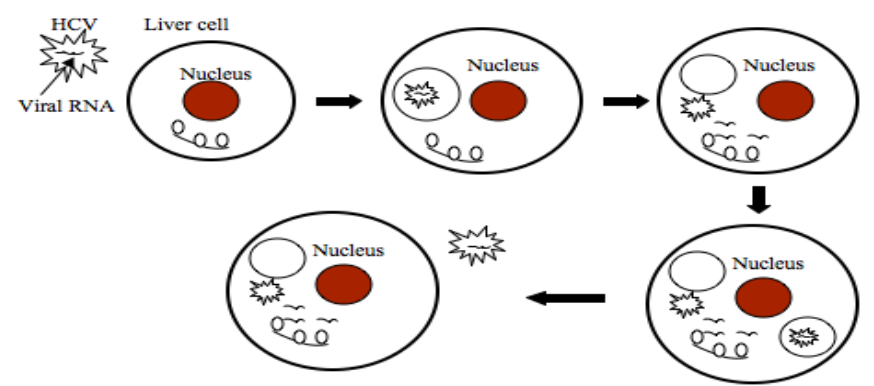

Figure 1. The sequence of biological events explaining how the virus enters a liver cell, makes numerous copies of RNA with the protein components manufactured by the ribosomes and, enter an empty vesicle to form complete virions then releasing new virions to infect other cells (Adapted:

http://www.hepccenter.org/hepcvirus.php) 


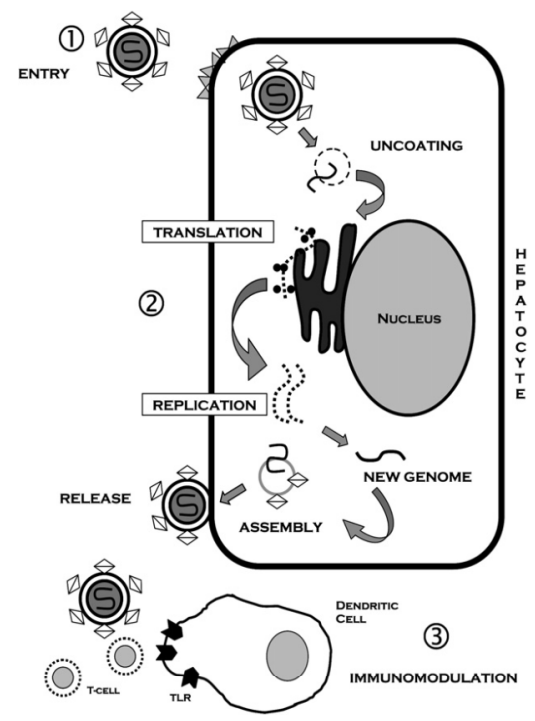

Figure 2. Hepatitis C virus life cycle (Source: Firpi \& Nelson, 2007, p. 684)

However, once infected, the cell death rate exhibits large lifespan variation with half-life between 1.7 and 70 days. This implies that early monitoring of viral load is essential to control HCV infection through faster killing of infected cells with IFN- $\alpha$ doses of 10 and 15 million international units (mIU) daily; standard current dose is between 3 to $15 \mathrm{mIU}$ (Neumann et al., 1998). Soriano et al. (2008) also presented that the fragile nature and short turnover of HCV-RNA molecules served as an opportunity for early eradication.

Table 3. The viral dynamic features of HCV. (Source: Soriano et al. 2008)

\begin{tabular}{ll}
\hline HCV & Dynamic features \\
\hline Virus & \\
Daily production of virions per day & $10^{12}$ \\
Half-life of free virions & $2-3$ hours \\
Half-life of intracellular virions & Hours (not dependent on infected cells $t_{1 / 2}$ ) \\
Mutation rate & Very high \\
Immune-mediated escape mutants & Frequent \\
Target Cells & \\
Half-life of infected cells & $1.7-70$ days (weeks) \\
Size of susceptible cells compartment & Probably large \\
Intracellular viral reservoir & No \\
\hline &
\end{tabular}

Chronic HCV infection is considered to be the main cause of chronic liver disease (Table 4), which, eventually evolves to cirrhosis or primary hepatocellular carcinoma (HCC), progressing to patients needing liver transplantation or even death (Dahari et al., 2005). HCV is described to be a clinically silent disease because 15 $-30 \%$ of infected are asymptomatic patients and has an alarming prevalence of $2-15 \%$ throughout the world (Neumann et al., 1998). The transmission of HCV is commonly via unscreened blood transfusion, direct contact with infected blood, unsafe sharing of needles and syringes and perinatal transmission.

Table 4. The risk of developing chronic infection, liver disease, cirrhosis and death in every 100 infected individuals. (Source: CDC, 2009)

\begin{tabular}{cl}
\hline \multicolumn{2}{c}{ Of every 100 persons infected with HCV: } \\
\hline $75-85$ & Develop chronic infection \\
$60-70$ & Develop chronic liver disease \\
$5-20$ & Develop cirrhosis over a period of 20-30 years \\
$1-5$ & Die from the consequences of chronic infection \\
\hline
\end{tabular}


A vaccine against $\mathrm{HCV}$ infection is still currently unavailable. However, a combined standard treatment with interferon - $\alpha$ (IFN) and ribavirin has shown an almost 50\% rate of sustained viral response (SVR) in the chronic phase (Reluga, Dahari \& Perelson, 2009) and, 100\% rate when treated with IFN in early initiation (89 days) after infection (Dahari et al., 2005).

According to Dahari et al. (2007), a typical therapy response should show a rapid viral decline at the start followed by a second slower decline until the virus becomes undetectable. A triphasic decline is also observed in some patients that indicated a promising initial rapid decline in viral load, followed by "shoulder phase" (slow or remains constant), and final phase of resumed viral decay (Dahari et al., 2007). However, upon therapy cessation, approximately $50 \%$ of treated HCV patients exhibited a rebound of viral plateau, to pretreatment levels that define whether, the antiviral therapy is only partially effective during treatment phase or will it be successful in eradicating HCV.

Therefore the aim of this study is to undertake a mathematical analysis of the stability of the classical mathematical model of HCV dynamics in order to reveal significant insights of HCV pathogenesis and dynamics. The methodology allows us to use similar techniques to model and study other infections. Hence, mathematical models are developed to study the kinetics and dynamics of HCV and evaluating the effectiveness of antiviral therapy. We then characterise the stability of the steady states of the mathematical model thereby offering a more complete understanding of the HCV dynamics. Our studies focus on the original model of HCV infection under therapy (Neumann et al., 1998), where we present results for the stability of both the uninfected and infected steady states, and finally compare the behaviour of the original model to the extended model (Dahari et al., 2007) that account for the ultimate $\mathrm{HCV}$ elimination. In order to accomplish these goals, the models and their parameters are presented and described, followed by linear stability analysis of the models to examine the asymptotic stability of each their steady states.

\section{Mathematical Model and Analysis of Hepatitis C Virus}

Numerous mathematical models describing the temporal dynamics of HCV have been proposed (Guedj \& Neumann, 2010; Chatterjee, Guedj \& Perelson, 2012; Rong \& Perelson, 2013). In all these studies, mathematical modelling plays a pivotal role in understanding and quantifying the biological mechanisms that govern HCV dynamics with or without therapy. For example, mathematical modelling has been shown to play a key role in highlighting the significance of obtaining frequent viral load measurements during treatment and has provided efficient tools for early prediction and the effects of therapeutic drugs on patient treatment (Mihm et al., 2006; Guedj et al., 2010; Guedj et al., 2013). Given the recent surge in the development of new direct acting antivirals agents for $\mathrm{HCV}$ therapy, mathematical modelling of viral kinetics under treatment continues to play an instrumental role in improving our knowledge and understanding of virus pathogenesis and in guiding drug development (Guedj et al., 2010; Chatterjee et al., 2012; Rong \& Perelson, 2013). Our study complements substantially these studies by offering a new perspective and understanding of the dynamics of HCV through detailed linear stability analysis. Our study is inspired by the Neumann et al. (1998) model that considers both extracellular and intracellular levels of infection. The original model has since been extended to take into account the direct-acting antiviral agents against HCV (Guedj \& Neumann, 2010). In this study, we focus on the original model and incorporate drug effectiveness bases on pegylated interferon and ribavirin; we do not take into account direct-acting antiviral agents (Guedj \& Neumann, 2010).

To proceed, we assume that the uninfected target cells are produced at a rate s, die at constant rate d per cell. On the other hand, the target cells are infected with de novo infection rate constant of $\beta$ and the infected cells die at a constant rate of $\delta$ per cell. The hepatitis $\mathrm{C}$ virions are produced inside the infected cells at an average rate $p$ per infected cell and have a constant clearance rate $c$ per virion. Thereby, viral persistence will occur when rate of viral production $(p)$, de novo infection $(\beta)$, and production of target cells $(s)$ exceeds the clearance rate $(c)$, death rate of infected cells $(\delta)$ and target cells death rate $(d)$. In addition, the therapeutic effect of IFN treatment in this model involved blocking virions production (referred to as drug effectiveness) and reducing new infections which, are described in fractions $(1-\varepsilon)$ and $(1-\eta)$, respectively. 


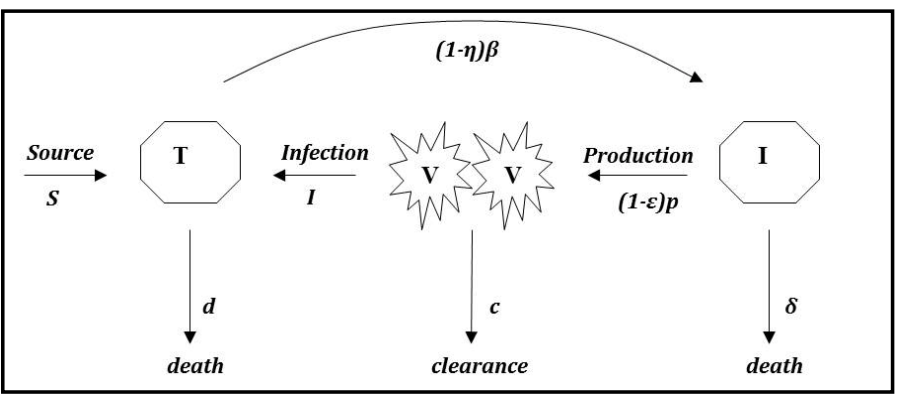

Figure 3. Schematic representation of the model of HCV infection. $T$ represents the target uninfected cells, $I$ is the infected cells and $V$ represents the free virus

\subsection{Mathematical Model of Hepatitis $C$}

The above assumptions lead to the following differential equations (Neumann et al., 1998):

$$
\begin{aligned}
& \frac{d T}{d t}=s-d T-(1-\eta) \beta V T, \\
& \frac{d I}{d t}=(1-\eta) \beta V T-\delta I, \\
& \frac{d V}{d t}=(1-\varepsilon) p I-c V,
\end{aligned}
$$

where the equations relate the dynamics relationship between, $T$ as the uninfected target cells (hepatocytes), $I$ as the infected cells and $V$ as the viral load (amount of viruses present in the blood). In this article, model system (1) - (3) is taken as the original model used to analyse the HCV dynamics.

\subsection{Stability Analyses}

The above model (1) - (3) predicts two possible steady states, one with no virus present (an uninfected steady state, $\mathrm{V}=\mathrm{I}=0$ ), and another with a constant level of virus (an endemically infected steady state). Thus, the equilibrium solutions of (1) - (3) must satisfy the following algebraic equations:

$$
\begin{aligned}
s-d T-(1-\eta) \beta V T & =0, \\
(1-\eta) \beta V T-\delta I & =0, \\
(1-\varepsilon) p I-c V & =0 .
\end{aligned}
$$

In the disease free equilibrium $(V=I=0)$ the population of the uninfected hepatocytes is derived as $\bar{T}_{0}=\frac{s}{d}$, where $\bar{T}_{0}$ also indicates complete viral eradication (cure) after the antiviral therapy (Dahari et al., 2007).

Here, if one assumes the target cell population remains constant during the course of the antiviral therapy, then the pre-treatment $\left(\varepsilon=\eta=0\right.$ ) quasi-steady state level of infected cells are derived from setting $\frac{d I}{d t}=0$ and $\frac{d V}{d t}=0 \quad$ in equations (2) and (3) (Neumann et al., 1998). This gives

$$
I_{0}=\frac{c V_{0}}{p} \quad T_{0}=\frac{c \delta}{p \beta},
$$

where $T_{0}$ represents the baseline target cell level and $V_{0}$ represents any given baseline pre-therapy HCV level.

Before therapy, $\mathcal{\varepsilon}=\eta=0$ and constant target cells.

Workings:

(3): $\quad p I=c V \Rightarrow \quad I_{0}=\frac{c V_{0}}{p}$

(2): $\quad \beta \mathrm{VT}-\delta \mathrm{I}=0 \Rightarrow \beta V T-\delta \frac{c V}{p}=0$, 


$$
\begin{gathered}
\mathrm{V}\left(\beta \mathrm{T}-\frac{\delta c}{\mathrm{p}}\right)=0, \\
\therefore V=0 \quad \text { or } \quad p \beta T_{0}=c \delta .
\end{gathered}
$$

(1): $s-d T-\beta V T=0 \Rightarrow \beta V \frac{c \delta}{p \beta}=\frac{s p \beta-d c \delta}{p \beta}$,

$$
\therefore V_{0}=\frac{p s}{c \delta}-\frac{d}{\beta} \text {. }
$$

Thus, the full steady state solution is further obtained by setting $\frac{d T}{d t}=0$ giving

$$
T_{0}=\frac{c \delta}{p \beta}, \quad I_{0}=\frac{s}{\delta}-\frac{d c}{p \beta}, \quad V_{0}=\frac{s p}{c \delta}-\frac{d}{\beta},
$$

where $T_{0}=\frac{c \delta}{p \beta}$ is the population of uninfected hepatocytes in the chronic infection prior to antiviral treatment. Now, it can be seen that the equilibrium points during antiviral therapy satisfy the following relations in the original model (1) - (3):

$$
\text { when } 0<\varepsilon<1 \text { and } 0<\eta<1 \text {, }
$$

Workings:

$$
\begin{gathered}
(1-\eta) \beta V T-\delta I=0 \quad \Rightarrow \quad(1-\eta) \beta V T-\delta \frac{c V}{p(1-\varepsilon)}=0, \\
V\left[(1-\eta) \beta T-\frac{c \delta}{p(1-\varepsilon)}\right]=0, \\
\therefore V=0 \quad \text { or } \quad T^{*}=\frac{c \delta}{(1-\eta) \boldsymbol{\beta}(1-\varepsilon) p} .
\end{gathered}
$$

$$
\begin{aligned}
& s-d T-(1-\eta) \beta V T=0 \\
& \Rightarrow \quad s-d \frac{\delta c}{(1-\eta) \beta(1-\varepsilon) p}=(1-\eta) \beta V \frac{\delta c}{(1-\eta) \beta(1-\varepsilon) p}, \\
& \therefore \quad V^{*}=\frac{(1-\varepsilon)(1-\eta) s p \boldsymbol{\beta}-\boldsymbol{d c} \delta}{(1-\eta) \delta c \boldsymbol{\beta}} .
\end{aligned}
$$

If $V^{*}=0$, the following uninfected steady state solution

$$
U^{*}\left(T^{*}, I^{*}, V^{*}\right)=\left(\frac{c \delta}{(1-\eta) \beta(1-\varepsilon) p}, 0,0\right)
$$

is obtained in which there is no infection. As a result, $T^{*}=\frac{\boldsymbol{c} \delta}{(\mathbf{1} \boldsymbol{\eta}) \boldsymbol{\beta}(\mathbf{1 - \varepsilon ) \boldsymbol { p }}}$ is assumed to be the number of liver cells (hepatocytes) present in a recovered patient, with effective drug efficacy and complete viral eradication during therapy.

If $V^{*} \neq 0$, the following infected steady state solution

$$
I_{F}^{*}\left(T^{*}, I^{*}, V^{*}\right)=\left(\frac{c \delta}{(1-\eta) \beta(1-\varepsilon) p}, \frac{(1-\varepsilon)(1-\eta) s p \beta-d c \delta}{(1-\eta)(1-\varepsilon) p \beta \delta}, \frac{(1-\varepsilon)(1-\eta) s p \beta-d c \delta}{(1-\eta) \delta c \beta}\right)
$$

is obtained where, the infection is termed endemic and analysis of the two steady states will exhibit a transcritical bifurcation point, that separates the regions of stability of the uninfected and infected steady states (Dahari et al., 2007). 
Since the model (1) - (3) predicts two steady states: the uninfected and the infected steady states, then the local stability of the steady states can be determined by linearising the non-linear equations of the model (1) - (3) around each steady state, and examining the corresponding eigenvalues of the characteristic equations (Dahari et al., 2007). The analysis of these eigenvalues which, will be discussed later, will indicate that there exists a bifurcation point at $(1-\eta)(1-\varepsilon)=\frac{c d \delta}{s p \beta}$. This bifurcation point will separate the regions of stability for the uninfected and the infected steady states. Thus, for a simple assumption that in the case of successful drug therapy, the viral load should approach zero, such that $0<\frac{(1-\eta)(1-\varepsilon) s p \beta}{c d \delta}<1$.

Theorem 1. Let $R_{0}$ be given by

$$
R_{0} \equiv \frac{s p \beta}{c d \delta}
$$

If $R_{0} \leq 1$, then $U^{*}=\left(\frac{c \delta}{(1-\eta) \beta(1-\varepsilon) p}, 0,0\right)$ is the only equilibrium point and is stable to small perturbations. If $R_{0}>1$, then the endemically infected equilibrium $I_{F} *=\left(\frac{c \delta}{(1-\eta) \beta(1-\varepsilon) p}, \frac{(1-\varepsilon)(1-\eta) s p \beta-d c \delta}{(1-\eta)(1-\varepsilon) p \beta \delta}, \frac{(1-\varepsilon)(1-\eta) s p \beta-d c \delta}{(1-\eta) \delta c \beta}\right)$ is asymptotically stable. The threshold parameter $\mathrm{R}_{0}$ is called the basic reproduction rate of the infection (Avendaño et al., 2002).

In order to prove Theorem 1, several different infection scenarios are investigated: infection may fade out without being established (disease-free); infection may spread with limited success (partial infection); or viral eradication with effective drug efficacy. Therefore, the analysis of the dynamics of the original model is further discussed corresponding to these scenarios, under the following conditions:

a) Disease-free equilibrium, $I=V=0$ requiring no therapy $\eta=\varepsilon=0$.

b) Chronic infection before therapy, $\eta=\varepsilon=0$.

c) Spontaneous cure, $I=V=0$, before treatment $(\varepsilon=\eta=0)$ or during therapy $(0<\varepsilon<1$, and $0<\eta<1)$.

d) Partial infection during therapy, $0<\varepsilon<1,0<\eta<1$.

e) $100 \%$ drug effectiveness $(\varepsilon=\eta=1)$ for viral eradication, $I=V=0$.

\section{Proof of Theorem 1.}

(a) Stability of the disease-free equilibrium $(I=V=0)$ requiring no antiviral therapy $(\eta=\varepsilon=0)$.

Since the antiviral drugs are not administered in a healthy individual with no HCV infection, $I=V=0$ (disease-free), the drug efficacy is considered to be zero $(\varepsilon=\eta=0)$. In this case, the original model (1) - (3) admits the uninfected steady state where the population of hepatocytes in the uninfected steady state in a healthy individual is $\bar{T}_{0}=\frac{s}{d}$. This gives the uninfected steady state before therapy as $\bar{U}_{0}\left(T^{*}, I^{*}, V^{*}\right)=\left(\frac{s}{d}, 0,0\right)$.

By linearising the non-linear equations of model (1) - (3), the Jacobian matrix $J(T, I, V)$ of the system (1) - (3) is given by

$$
J(T, I, V)=\left[\begin{array}{ccc}
-d-(1-\eta) \beta V & 0 & -(1-\eta) \beta T \\
(1-\eta) \beta V & -\delta & (1-\eta) \beta T \\
0 & (1-\varepsilon) p & -c
\end{array}\right]
$$

Firstly, the local stability of uninfected steady state before therapy initiation, $\bar{U}_{0}\left(T^{*}, I^{*}, V^{*}\right)=\left(\frac{s}{d}, 0,0\right)$ is governed by the eigenvalues of the matrix 


$$
J\left(\bar{U}_{0}\right)=\left[\begin{array}{ccc}
-d & 0 & -\frac{\beta s}{d} \\
0 & -\delta & \frac{\beta s}{d} \\
0 & p & -c
\end{array}\right]
$$

which gives $\lambda_{1}=-d$ and the other two roots are solutions of the quadratic equation

$$
\begin{aligned}
& \lambda^{2}+(\delta+c) \lambda+\delta c-\frac{\beta s p}{d}=0, \\
& \therefore \lambda_{2,3}=\frac{-(\delta+c) \pm \sqrt{(\delta+c)^{2}-4\left(\delta c-\frac{\beta s p}{d}\right)}}{2} .
\end{aligned}
$$

Lemma 2. The Routh-Hurwitz criterion (Kot, 2001; Murray, 2002) states that a necessary and sufficient condition that the equation

$$
x^{n}+a_{1} x^{n-1}+\ldots+a_{n}=0,
$$

(with real coefficients) have only roots of negative real part if the values of the determinants of the matrices are all positive,

$$
\begin{aligned}
D_{1}=a_{1}>0, & D_{2}=\left[\begin{array}{cc}
a_{1} & a_{3} \\
1 & a_{2}
\end{array}\right]>0, \\
D_{k} & =\left[\begin{array}{cccccc}
a_{1} & a_{3} & . & . & . & \cdot \\
1 & a_{2} & a_{4} & . & . & . \\
0 & a_{1} & a_{3} & . & . & . \\
0 & 1 & a_{2} & . & . & . \\
. & . & . & . & . & . \\
0 & 0 & . & . & . & a_{k}
\end{array}\right]>0
\end{aligned}
$$

For quadratic and cubic polynomials, these conditions reduce to
$n=2$,
$a_{1}>0$,
$a_{2}>0$
$n=3$,
$a_{1}>0$,
$a_{2}>0$,
$a_{1} a_{2}>a_{3}$.

Therefore for equation (8), if $a_{l}$ and $a_{2}$ are both positive, then applying the Routh-Hurwitz criterion (Lemma 2) to this quadratic equation guarantees the eigenvalues to have negative real part, and that two conditions must be satisfied for asymptotic stability of the endemic equilibrium:

(1) $\quad a_{1}>0, \quad(\delta+c)>0, \quad$ (satisfied)

(2) $\quad a_{2}>0, \quad \delta c-\frac{\beta s p}{d}>0$ (satisfied if and only if $\frac{\beta s p}{d \delta c}<1$ )

Similarly, the equilibrium of a model that consists of a system of ordinary differential equation is considered asymptotically stable if all the eigenvalues of the characteristic equation have negative real part (Brauer, 2004). Thus for stability, this occurs if and only if the coefficients of (8) are positive, which hold true provided

$$
\frac{\beta s p}{d}<\delta c \quad \Rightarrow \quad \frac{\beta s p}{d \delta c}<1 \quad \Rightarrow \quad R_{0}<1 .
$$

Therefore, the disease-free steady state $\bar{U}_{0}=\left(\frac{s}{d}, 0,0\right)$ is locally asymptotically stable for $R_{0}<1$. This proves the first part of Theorem 1. If $R_{0}>1$, then $\bar{U}_{0}=\left(\frac{s}{d}, 0,0\right)$ becomes an unstable critical point. This means that for HCV to infect the liver, $R_{0}$ must be greater than 1, indicating that once a cell become infected, it can cause more than one uninfected cell to be infected. Also if $R_{0}>1$, the HCV infects new cells $(\beta s p)$ faster than infected cells die $(\delta d)$ or being cleared $(c)$, thus Theorem $1\left(R_{0}>1\right)$ may correspond to partial infection of the liver.

(b) Stability of the chronically infected steady state before therapy, $\eta=\varepsilon=0$. 
Since HCV infection is sometimes symptomless, there is a possibility that an infected individual might not seek treatment until the chronic stage. In this case, the infected steady state solution before antiviral therapy is given by,

$$
T_{0}=\frac{c \delta}{\beta p} \quad I_{0}=\frac{s p \beta-d c \delta}{p \beta \delta} \quad \text { and } \quad V_{0}=\frac{s p \beta-d c \delta}{\delta c \beta}
$$

such that, $I_{F}\left(T_{0}, I_{0}, V_{0}\right)=\left(\frac{c \delta}{\beta p}, \frac{s p \beta-d c \delta}{p \beta \delta}, \frac{s p \beta-d c \delta}{\delta c \beta}\right)$.

Now, the local stability of the infected steady state, $I_{F}=\left(\frac{c \delta}{\beta p}, \frac{s p \beta-d c \delta}{p \beta \delta}, \frac{s p \beta-d c \delta}{\delta c \beta}\right)$ is governed by the eigenvalues of the matrix

$$
J\left(I_{F}\right)=\left[\begin{array}{ccc}
-2 d-\frac{s p \beta}{\delta c} & 0 & -\frac{c \delta}{p} \\
\frac{s p \beta-d c \delta}{\delta c} & -\delta & \frac{c \delta}{p} \\
0 & p & -c
\end{array}\right]
$$

The characteristic equation of the linearised system is given by the following cubic equation:

$$
\begin{gathered}
F(\lambda)=-\lambda^{3}-\left[2 d+\frac{s p \beta}{\delta c}+(\delta+c)\right] \lambda^{2}-\left[2 d(\delta+c)+\frac{s p \beta}{\delta c}(\delta+c)\right] \lambda+d c \delta-s p \beta \\
F(\lambda)=\lambda^{3}+a_{1} \lambda^{2}+a_{2} \lambda+a_{3}
\end{gathered}
$$

with coefficients given by

$$
\begin{gathered}
a_{1}=\left[2 d+\frac{s p \beta}{\delta c}+(\delta+c)\right], \\
a_{2}=\left[2 d(\delta+c)+\frac{s p \beta}{\delta c}(\delta+c)\right], \\
a_{3}=s p \beta-d c \delta .
\end{gathered}
$$

If $a_{1}, a_{2}$, and $a_{3}$ are all positive, then applying the Routh-Hurwitz criterion (Lemma 2) to this cubic equation guarantees the eigenvalues to have negative real part, and that three conditions must be satisfied for asymptotic stability of the endemic equilibrium:

$$
\begin{aligned}
& \text { (1) } a_{1}>0, \quad\left[2 d+\frac{s p \beta}{\delta c}+(\delta+c)\right]>0, \\
& \text { (2) } \quad a_{2}>0, \quad\left[2 d(\delta+c)+\frac{s p \beta}{\delta c}(\delta+c)\right]>0, \\
& \text { (3) } a_{1} a_{2}>a_{3}, \quad\left[2 d+\frac{s p \beta}{\delta c}+(\delta+c)\right]\left[2 d(\delta+c)+\frac{s p \beta}{\delta c}(\delta+c)\right]>s p \beta-d c \delta \text {, } \\
& \Rightarrow \frac{\left[2 d+\frac{s p \beta}{\delta c}+(\delta+c)\right]\left[2 d(\delta+c)+\frac{s p \beta}{\delta c}(\delta+c)\right]}{d c \delta}>\frac{s p \beta}{d c \delta}-1 .
\end{aligned}
$$

All three conditions are satisfied if and only if $\frac{s p \beta}{d c \delta}>1$ for asymptotic stability of the chronically infected steady state. The first and second conditions are satisfied such that $d$ is always greater than zero and $(\delta+c)$ is always positive. Thus, the third condition implies for asymptotic stability if, and only if $R_{0}>1$. This further proves second part of Theorem 1 . The stability of the chronically infected steady state is interpreted medically as the symptomless of the infection which is the silent killer of the disease.

(c) Stability of spontaneous cure, $I=V=0$, before treatment $(\varepsilon=\eta=0)$ or during therapy

$$
(0<\varepsilon<1 \text { and } 0<\eta<1) \text {. }
$$


Assuming that spontaneous cure is available, such that the infected cells and virions are successfully eradicated, where $I=V=0$. The terms spontaneous regression or even spontaneous cure is commonly observed in cancer suffering patients where, they experienced an unexpected transient or final improvement from the disease. This was documented by Cole and Everson (1966): "The partial or complete disappearance of a malignant tumour in the absence of all treatment, or in the presence of therapy which is considered inadequate to exert significant influence on neoplastic disease." In similar context, spontaneous cure in HCV infection is recognised as infection that fades out in the absence of treatment or, during therapy to give the steady states,

$U_{0}^{*}=\left(\frac{c \delta}{\beta p}, 0,0\right)$ and $U^{*}=\left(\frac{c \delta}{(1-\eta) \beta(1-\varepsilon) p}, 0,0\right)$ respectively. Since, the Jacobian matrix $J\left(T^{*}, I^{*}, V^{*}\right)$ of the system (1)-(3) is given by

$$
J\left(T^{*}, I^{*}, V^{*}\right)=\left[\begin{array}{ccc}
-d-(1-\eta) \beta V & 0 & -(1-\eta) \beta T \\
(1-\eta) \beta V & -\delta & (1-\eta) \beta T \\
0 & (1-\varepsilon) p & -c
\end{array}\right] .
$$

Firstly, the local stability of spontaneous cure before therapy is $U_{0}^{*}=\left(\frac{c \delta}{\beta p}, 0,0\right)$ is governed by the eigenvalues of the matrix

$$
J\left(U^{*}\right)=\left[\begin{array}{ccc}
-d & 0 & -\frac{c \delta}{p} \\
0 & -\delta & \frac{c \delta}{p} \\
0 & p & -c
\end{array}\right]
$$

which are given by $\lambda_{1}=-d$ and the other two are roots of the quadratic equation

$$
\begin{aligned}
& \lambda^{2}+(\delta+c) \lambda=0 \\
& \text { i.e. } \quad \lambda_{2}=0 \quad \text { or } \quad \lambda_{3}=-(\delta+c) \text {. }
\end{aligned}
$$

Secondly, the local stability of spontaneous cure during therapy is $U^{*}=\left(\frac{c \delta}{(1-\eta) \beta(1-\varepsilon) p}, 0,0\right)$ is governed by the eigenvalues of the matrix

$$
J\left(U^{*}\right)=\left[\begin{array}{ccc}
-d & 0 & -\frac{c \delta}{(1-\varepsilon) p} \\
0 & -\delta & \frac{c \delta}{(1-\varepsilon) p} \\
0 & (1-\varepsilon) p & -c
\end{array}\right]
$$

which are given by $\lambda_{1}=-d$ and the other two are roots of the quadratic equation

$$
\begin{gathered}
\lambda^{2}+(\delta+c) \lambda=0 \\
\text { i.e. } \quad \lambda_{2}=0 \quad \text { or } \quad \lambda_{3}=-(\delta+c) \text {. }
\end{gathered}
$$

Theorem 3. Consider the system $\dot{x_{1}}=f_{1}\left(\lambda, x_{1}, x_{2}\right)$ and $\dot{x_{2}}=-x_{2}+f_{2}\left(\lambda, x_{1}, x_{2}\right)$ in the case where $\lambda$ is a scalar parameter. If $\frac{d f_{1}}{d \lambda}(0,0,0) \neq 0, \frac{d^{2} f_{1}}{d x_{1}^{2}}(0,0,0) \neq 0$ then there is a saddle-node bifurcation at $\lambda=0$, that is when $\lambda \frac{d f_{1}}{d \lambda} \frac{d^{2} f_{1}}{d x_{1}^{2}}<0$, there are two hyperbolic equilibria, one saddle and the other an asymptotically stable node, and no equilibrium when $\lambda \frac{d f_{1}}{d \lambda} \frac{d^{2} f_{1}}{d x_{1}^{2}}>0$ (Hale \& Kocak, 1991). 
Using Hale and Kocak (1991) Theorem 3, both the steady states of spontaneous cure before and during therapy, that is $U_{0}^{*}=\left(\frac{c \delta}{\beta p}, 0,0\right)$ and $U^{*}=\left(\frac{c \delta}{(1-\eta) \beta(1-\varepsilon) p}, 0,0\right)$ respectively, have no equilibrium because $\frac{c \delta}{\beta p} \frac{d T}{d t} \frac{d^{2} T}{d t^{2}} \quad$ is always greater zero.

(d) Stability of the partial infected steady state during therapy $0<\varepsilon<1,0<\eta<1$.

During the chronic stage of the infection, patients will be administered antiviral therapy $(0<\varepsilon<1$ and $0<\eta<1)$, and hepatocytes will be partially infected. Since, the linearised Jacobian matrix of model (1) - (3) is evaluated as

$$
\left[\begin{array}{ccc}
-d-(1-\eta) \beta V & 0 & -(1-\eta) \beta T \\
(1-\eta) \beta V & -\delta & (1-\eta) \beta T \\
0 & (1-\varepsilon) p & -c
\end{array}\right]
$$

For $I_{F}^{*}\left(T^{*}, I^{*}, V^{*}\right)=\left(\frac{c \delta}{(1-\eta) \beta(1-\varepsilon) p}, \frac{(1-\varepsilon)(1-\eta) s p \beta-d c \delta}{(1-\eta)(1-\varepsilon) p \beta \delta}, \frac{(1-\varepsilon)(1-\eta) s p \beta-d c \delta}{(1-\eta) \delta c \beta}\right)$ that can be written as

$$
J\left(I_{F}^{*}\right)=\left[\begin{array}{ccc}
-d-\frac{(1-\varepsilon)(1-\eta) s p \beta-d c \delta}{\delta c} & 0 & -\frac{c \delta}{(1-\varepsilon) p} \\
\frac{(1-\varepsilon)(1-\eta) s p \beta-d c \delta}{\delta c} & -\delta & \frac{c \delta}{(1-\varepsilon) p} \\
0 & (1-\varepsilon) p & -c
\end{array}\right]
$$

The characteristic equation of the linearised system is given by the following cubic equation:

$$
\begin{gathered}
F(\lambda)=-\lambda^{3}-[d+M+(\delta+c)] \lambda^{2}-[d(\delta+c)+M(\delta+c)] \lambda+d c \delta-(1-\varepsilon)(1-\eta) \operatorname{sp} \beta \\
F(\lambda)=\lambda^{3}+a_{1} \lambda^{2}+a_{2} \lambda+a_{3}
\end{gathered}
$$

with $M=\frac{(1-\varepsilon)(1-\eta) s p \beta-d c \delta}{\delta c}$. The coefficients are given by

$$
\begin{gathered}
a_{1}=\left[d+\frac{(1-\varepsilon)(1-\eta) s p \beta-d c \delta}{\delta c}+(\delta+c)\right], \\
a_{2}=\left[d(\delta+c)+\frac{(1-\varepsilon)(1-\eta) s p \beta-d c \delta}{\delta c}(\delta+c)\right], \\
a_{3}=(1-\varepsilon)(1-\eta) s p \beta-d c \delta .
\end{gathered}
$$

If $a_{1}, a_{2}$ and $a_{3}$ are all positive, then applying the Routh-Hurwitz criterion (Lemma 2) to this cubic equation guarantees the eigenvalues to have negative real part, and that two conditions must be satisfied for asymptotic stability of the endemic equilibrium:

(1) $a_{1}>0$,

$$
\left[d+\frac{(1-\varepsilon)(1-\eta) s p \beta-d c \delta}{\delta c}+(\delta+c)\right]>0
$$

(2) $a_{2}>0$,

$$
\left[d(\delta+c)+\frac{(1-\varepsilon)(1-\eta) s p \beta-d c \delta}{\delta c}(\delta+c)\right]>0
$$

(3)

$$
\begin{aligned}
& {\left[d+\frac{(1-\varepsilon)(1-\eta) s p \beta-d c \delta}{\delta c}+(\delta+c)\right]\left[d(\delta+c)+\frac{(1-\varepsilon)(1-\eta) s p \beta-d c \delta}{\delta c}(\delta+c)\right]>(1-\varepsilon)(1-\eta) s p \beta-d c \delta} \\
& \Rightarrow \frac{\left[d+\frac{(1-\varepsilon)(1-\eta) s p \beta-d c \delta}{\delta c}+(\delta+c)\right]\left[d(\delta+c)+\frac{(1-\varepsilon)(1-\eta) s p \beta-d c \delta}{\delta c}(\delta+c)\right]}{d c \delta}>\frac{(1-\varepsilon)(1-\eta) s p \beta}{d c \delta}-1
\end{aligned}
$$

All three conditions are satisfied if, and only if $\frac{(1-\eta)(1-\varepsilon) s p \beta}{d c \delta}>1$ for asymptotic stability of partial infected 
steady state. This further proves the earlier assumption that for successful drug therapy, the viral load should approach zero, such that $0<\frac{(1-\eta)(1-\varepsilon) \operatorname{sp} \beta}{c d \delta}<1$.

(e) $100 \%$ drug effectiveness $(\varepsilon=\eta=1)$ for viral eradication, $I=V=0$.

If the efficacy of the drug is assumed to be $100 \%(\varepsilon=\eta=1)$, then viral eradication $(I=V=0)$ should follow to clear the infection. The assumption leads to the model (1) - (3) differential equations reducing to

$$
\frac{d T}{d t}=s-d T \quad \Rightarrow \quad T=\frac{s}{d}+\left[T\left(t_{0}\right)-\frac{s}{d}\right] e^{-d\left(t-t_{0}\right)}
$$

For $d>0$, then as $t \rightarrow \infty, T \rightarrow \frac{s}{d}$. This equals the disease-free equilibrium. This is termed as medicinal cure.

In summary, for model (1) - (3), the stability of the uninfected and infected steady states were determined by the Jacobian matrix, which for the first steady state, that is the disease-free steady state is given by

$$
\bar{U}_{0}\left(T^{*}, I^{*}, V^{*}\right)=\left(\frac{s}{d}, 0,0\right) \quad \text { is }\left\{\begin{array} { c } 
{ \text { stable } } \\
{ \text { unstable } }
\end{array} \text { if } \left\{\begin{array}{l}
R_{0}<1 \\
R_{0}>1
\end{array}\right.\right.
$$

Similarly, for the second steady state, the chronically infected state before therapy,

$$
I_{F}=\left(\frac{c \delta}{\beta p}, \frac{s p \beta-d c \delta}{p \beta \delta}, \frac{s p \beta-d c \delta}{\delta c \beta}\right) \text { is }\left\{\begin{array} { c } 
{ \text { stable } } \\
{ \text { unstable } }
\end{array} \text { if } \left\{\begin{array}{l}
R_{0}>1 \\
R_{0}<1
\end{array}\right.\right.
$$

Finally, for the infected steady state during therapy,

$$
I_{F}^{*}=\left(\frac{c \delta}{(1-\eta) \beta(1-\varepsilon) p}, \frac{(1-\varepsilon)(1-\eta) s p \beta-d c \delta}{(1-\eta)(1-\varepsilon) p \beta \delta}, \frac{(1-\varepsilon)(1-\eta) s p \beta-d c \delta}{(1-\eta) \delta c \beta}\right) \text { is }\left\{\begin{array} { c } 
{ \text { stable } } \\
{ \text { unstable } }
\end{array} \text { if } \left\{\begin{array}{c}
(1-\eta)(1-\varepsilon) R_{0}>1 \\
0<(1-\eta)(1-\varepsilon) R_{0}<1 .
\end{array}\right.\right.
$$

Now, let us consider the physiological implications of these results. In the case that $R_{0}<1$, there is only one stable steady state where only disease-free individuals exist. In terms of the original parameters from (7), this corresponds to $\operatorname{sp} \beta<c d \delta$. For example, if $p$ (viral production), $c$ (clearance rate of infected cells), $\beta$ (de novo infection), and $\delta$ (death rate of infected cells) are approximately the same, then there will be no competition between the uninfected and infected cells.

In the case where $R_{0}>1$, if the production of uninfected and infected cells is always greater than the death rate of uninfected and infected cells, then the competition is such that the two infected steady states can exist. Thus, the ultimate viral eradication (neither stable nor unstable) will depend crucially on the starting advantage each cell has.

\section{The Extended Model}

As mentioned earlier, the original model of HCV infection proposed by Neumann et al. (1998) disregards the proliferation of both infected and uninfected cells and, its solutions did not predict the triphasic viral decay observed in some chronic patients. Therefore, we will look into another model by Dahari et al. (2007), which is termed the extended model. This model expands on the original HCV viral-dynamic model by incorporating density-dependent proliferation $(r)$ that allows growth of liver to a maximum size, $T_{\max }$. The corresponding differential equation of the extended model is given by,

$$
\begin{gathered}
\frac{d T}{d t}=s+r T\left(1-\frac{T+I}{T_{\max }}\right)-d T-(1-\eta) \beta V T, \\
\frac{d I}{d t}=(1-\eta) \beta V T+r I\left(1-\frac{T+I}{T_{\max }}\right)-\delta I, \\
\frac{d V}{d t}=(1-\varepsilon) p I-c V,
\end{gathered}
$$

where uninfected hepatocytes $(T)$ and infected hepatocytes (I) can proliferate logistically with maximum proliferation rate $r$ up to the number of hepatocytes being less than $T_{\max }$. The remaining parameters are defined similarly to the original model and the estimated parameter ranges and units are shown in Table 5. The analysis of the extended model involves nondimensionalisation to reduce the number of parameters. 
Table 5. Estimated parameter ranges and units for HCV infection model (9) - (11). (Sources: Reluga et al., 2009 and Dahari et al., 2009)

\begin{tabular}{llll}
\hline Variables & \multirow{2}{*}{ Units } & \multicolumn{2}{c}{ Parameter ranges } \\
\cline { 3 - 4 } & & Reluga et al. (2009) & Dahari et al. (2009) \\
\cline { 3 - 4 }$s$ & cells ml $^{-1}$ day $^{-1}$ & $1.0-1.8 \times 10^{5}$ & $1<s \leq d T_{\max }$ \\
$r$ & day $^{-1}$ & $0.002-3.4$ & $1.0-3.0$ \\
$T_{\max }$ & cells ml $^{-1}$ & $4.6 \times 10^{6}-1.3 \times 10^{7}$ & $0.4 \times 10^{7}-1.3 \times 10^{7}$ \\
$d$ & day $^{-1}$ & $0.001-0.014$ & $0.001-0.014$ \\
$\beta$ & virus $^{-1} \mathrm{ml} \mathrm{day}$ & \\
$\delta$ & day $^{-1}$ & $10^{-8}-10^{-6}$ & $10^{-9}-10^{-5}$ \\
$\delta$ & $10^{-3}-0.5$ & $d \leq \delta \leq 0.5$ \\
$p$ & virus cell $^{-1}$ day $^{-1}$ & $0.1-44$ & $0.1-45$ \\
$c$ & day $^{-1}$ & $0.8-22$ & $0.8-22$ \\
\hline
\end{tabular}

According to Reluga et al. (2009), the viral dynamics model (9) - (11) shall decompose into two time scales: a fast time scale starting at $t_{0}$ where infected hepatocytes are relatively constant resulting in the following equation,

$$
V(t)=\frac{(1-\varepsilon) p}{c} I(t)+\left[V\left(t_{0}\right)-\frac{(1-\varepsilon) p}{c} I\left(t_{0}\right)\right] e^{-c\left(t-t_{0}\right)}
$$

and also a slow time scale where

$$
V(t) \approx \frac{(1-\varepsilon) p}{c} I(t)
$$

Before antiviral therapy being initiated, for a chronically infected patient where $I\left(t_{0}\right)=\frac{c V\left(t_{0}\right)}{p}$, equation (12) simplified to become

$$
V(t)=\frac{(1-\varepsilon) p}{c} I(t)+\left[\varepsilon V\left(t_{0}\right)\right] e^{-c\left(t-t_{0}\right)}
$$

Workings for equation (12):

$$
\begin{aligned}
& \frac{d V}{d t}=(1-\varepsilon) p I-c V, \\
& \frac{d V}{d t}+c V=(1-\varepsilon) p I .
\end{aligned}
$$

Multiplying by an integrating factor $e^{c t}$

$$
\begin{gathered}
e^{c t} \frac{d V}{d t}+e^{c t} c V=e^{c t}(1-\varepsilon) p I, \\
\frac{d}{d t}\left(e^{c t} V\right)=e^{c t}(1-\varepsilon) p I \\
V(t)=\frac{(1-\varepsilon) p}{c} I(t)+A e^{-c t}
\end{gathered}
$$

At $t_{0}$,

$$
\begin{gathered}
V\left(t_{0}\right)=\frac{(1-\varepsilon) p}{c} I\left(t_{0}\right)+A e^{-c t_{0},} \\
A=e^{c t_{0}}\left[V\left(t_{0}\right)-\frac{(1-\varepsilon) p}{c} I\left(t_{0}\right)\right], \\
\therefore V(t)=\frac{(1-\varepsilon) p}{c} I(t)+\left[V\left(t_{0}\right)-\frac{(1-\varepsilon) p}{c} I\left(t_{0}\right)\right] e^{-c\left(t-t_{0}\right) .}
\end{gathered}
$$

Now, the dynamics of the model (9) - (11) can be reduced to two equations by introducing the dimensionless 
state variables

$$
\tau=\hat{t} t \quad u=\hat{T} T \quad v=\hat{I} I
$$

and the dimensionless parameters

$$
r=\hat{t}, \quad a=\frac{s}{r T_{\max }}, \quad b=\frac{p \beta T_{\max }}{c r}, \quad g=\frac{d}{r}, \quad h=\frac{\delta}{r}, \quad(1-\alpha)=(1-\varepsilon)(1-\eta),
$$

such that on the time scales larger than $\frac{1}{c}$ where $V(t) \approx \frac{(1-\varepsilon) p}{c} I(t)$ we can approximate the dimensionless model as

$$
\begin{aligned}
& \frac{d u}{d \tau}=a+u(1-u-v)-g u-(1-\alpha) b u v=w(u, v) \\
& \frac{d v}{d \tau}=v(1-u-v)+(1-\alpha) b u v-h v=z(u, v)
\end{aligned}
$$

Workings for the dimensionless model based on time scales larger than $\frac{1}{c}$ such that $V(t) \approx \frac{(1-\varepsilon) p}{c} I(t)$ :

Let $\tau=\hat{t} t$

$$
u=\widehat{T} T \quad v=\hat{I I}
$$

$$
\therefore \quad \frac{d \tau}{d t}=\hat{t} \quad \frac{d u}{d \tau}=\hat{T} \frac{d T}{d \tau} \quad \frac{d v}{d \tau}=\hat{I} \frac{d I}{d \tau}
$$

$\Rightarrow \frac{d u}{d \tau}=\frac{\hat{T}}{\hat{t}}\left[s+r T\left(1-\frac{T+I}{T_{\max }}\right)-d T-(1-\eta) \beta V T\right]$

$$
\begin{aligned}
& =\frac{1}{\hat{t}}\left[s \hat{T}+r T \hat{T}\left(1-\frac{T \hat{T}}{\hat{T} T_{\max }}-\frac{I \hat{I}}{\hat{I} T_{\max }}\right)-d T \hat{T}-\frac{(1-\eta)(1-\varepsilon) \beta p I \hat{I} T \hat{T}]}{c \hat{I}}\right] \\
& =\frac{r}{\hat{t}}\left[\frac{s \hat{T}}{r}+T \hat{T}\left(1-\frac{T \hat{T}}{\hat{T} T_{\max }}-\frac{I \hat{I}}{\hat{I} T_{\max }}\right)-\frac{d T \hat{T}}{r}-\frac{(1-\eta)(1-\varepsilon) \beta p I \hat{I} T \hat{T}]}{r c \hat{I}}\right]
\end{aligned}
$$

Choosing the following dimensionless parameters

$\hat{T}=\hat{I}=\frac{1}{T_{\max }}, r=\hat{t}, a=\frac{s}{r T_{\max }}, \quad b=\frac{p \beta T_{\max }}{c r}, g=\frac{d}{r}, \quad h=\frac{\delta}{r} \quad,(1-\alpha)=(1-\varepsilon)(1-\eta)$,

we get,

$$
\frac{d u}{d \tau}=a+u(1-u-v)-(1-\alpha) b u v-g u
$$

Similarly,

$$
\begin{array}{r}
\frac{d v}{d \tau}=\frac{\hat{I}}{\hat{t}}\left[(1-\eta) \beta V T+r I\left(1-\frac{T+I}{T_{\max }}\right)-\delta I\right] \\
\frac{d v}{d \tau}=\frac{r}{\hat{t}}\left[I \hat{I}\left(1-\frac{T \hat{T}}{\hat{T} T_{\max }}-\frac{I \hat{I}}{\hat{I} T_{\max }}\right)+\frac{(1-\eta)(1-\varepsilon) \beta p I \hat{I} T \hat{T}}{r c T^{*}}-\frac{\delta I \hat{I}}{r}\right] \\
\therefore \frac{d v}{d \tau}=v(1-u-v)+(1-\alpha) b u v-h v
\end{array}
$$

\subsection{Steady States of the Extended Model}

Assuming there is no increase in uninfected hepatocytes through migration or differentiation $(\mathrm{a}=0)$ on the basis that liver damage occurs, the model (14) - (15) simplifies to

$$
\begin{gathered}
\frac{d u}{d \tau}=u(1-u-v)-g u-(1-\alpha) b u v=f(u, v) \\
\frac{d v}{d \tau}=v(1-u-v)+(1-\alpha) b u v-h v=g(u, v)
\end{gathered}
$$


The above model (16) - (17) can be used to study and understand the HCV dynamics in an infected individual as the ensuing dynamics depends on the relative parameter values. During pre-treatment $(\alpha=0)$, the dynamics described in model (16) - (17) is a modification of the Lokta-Volterra equation such that, the equilibrium or steady states are solutions of $\frac{d u}{d \tau}=f(u, v)=0$ and $\frac{d v}{d \tau}=g(u, v)=0$. When $f(u, v)=0$, the solutions are $u=0$ and $v=\frac{1-u-g}{1+b}$. While $g(u, v)=0$ we have the solutions $v=0$ and $u=\frac{1-v-h}{1-b}$. There are four steady states that can be derived from model (16)-(17) through the intersections of the $\frac{d u}{d \tau}=0 \quad$ and $\frac{d v}{d \tau}=0$ solutions. The intersections of the $\frac{d u}{d \tau}=\mathbf{0}$ and $\frac{d v}{d \tau}=\mathbf{0}$ are given by

$$
\begin{array}{lll}
u=0 & \text { and } & v=\frac{1-u-g}{1+b} \\
v=0 & \text { and } & u=\frac{1-v-h}{1-b}
\end{array}
$$

resulting in the following steady states,

$$
(0,0),(1-g, 0),(0,1-h),\left(\frac{h(1+b)-(b+g)}{b^{2}}, \frac{g(1-b)+(b-h)}{b^{2}}\right)
$$

The results from equations (16) and (17) are interpreted as liver-free solution (0,0), disease-free solution $(1-g, 0)$, total infection solution $(0,1-h)$ and partial-infection solution $\left(\frac{h(1+b)-(b+g)}{b^{2}}, \frac{g(1-b)+(b-h)}{b^{2}}\right)$.

Now, assuming that in a healthy individual, the liver is constantly producing uninfected hepatocytes such that $a \neq 0$ (migration occurs), then the steady states of equations (14) and (15) are solutions of $\frac{d u}{d \tau}=w(u, v)=0$ and $\frac{d v}{d \tau}=z(u, v)=0$. During pre-treatment $(\alpha=0)$, the $z(u, v)=0$ gives,

$$
v=0 \quad \text { and } \quad u=\frac{(1-h-v)}{(1-b)}
$$

The $w(u, v)=0$ gives only,

$$
v=\frac{a+u(1-g-u)}{u(1+b)}
$$

There are five steady states derived from the intersections of $\frac{d u}{d \tau}$ and $\frac{d v}{d \tau}$ solutions, $\left(\frac{1-h}{1-b}, 0\right)$, $\left(\frac{(1-g)+\sqrt{(g-1)^{2}+4 a}}{2}, 0\right), \quad\left(\frac{(1-g)-\sqrt{(g-1)^{2}+4 a}}{2}, 0\right), \quad$ and the other two steady states are $u=\frac{[(1-g)-(1-h)(1+b)] \pm \sqrt{[(1-g)-(1-h)(1+b)]^{2}+4 a b^{2}}}{2 b^{2}}$ with $v=(1-h)-(1-b) u$. In this case, the analysis of the steady states is best illustrated using phase portraits (a representative set of trajectories) to discuss the behaviour of the trajectories for stability.

\subsection{The Stability Analysis of the Extended Model}

Before we discuss the stability of the steady states, we will firstly look at the parameter ranges of values of model (14) - (15). From the results of the four steady states: liver-free solution $(0,0)$, disease-free solution $(1-g, 0)$, total infection solution $(0,1-h)$ and partial-infection solution $\left(\frac{h(1+b)-(b+g)}{b^{2}}, \frac{g(1-b)+(b-h)}{b^{2}}\right)$, it can 
be deduced that $0<\frac{b+g}{1+b}<h<b<1$. Thus, the ranges of values (Table 6 ) used for plotting the phase portraits have been estimated from the maximum and minimum of the original parameter values (Table 5). For any given value of $g, h$ is considered to be a critical value such that it will pre-determine the values of $b$ for $\frac{b+g}{1+b}<h<b$ (Table 7).

Table 6. The parameters ranges are calculated from the ranges of parameters values given in Table 5 . Note that the value of $r$ is taken to be the maximum i.e. $r=3.4$

\begin{tabular}{ll}
\hline Parameters & Parameter Ranges \\
\hline$g=\frac{d}{r}$ & $0.000294-0.00412$ \\
$h=\frac{\delta}{r}$ & $0.000294-0.1471$ \\
$b=\frac{p \beta T_{\max }}{c r}$ & $0.0000615-210.294$ \\
$\frac{b+g}{1+b}$ & $0.00000168-210.285$ \\
\hline
\end{tabular}

Table 7. The parameters range of values for plotting phase portraits of equations (16) and (17)

\begin{tabular}{lcc}
\hline \multirow{3}{*}{ Parameters } & \multicolumn{3}{c}{$\frac{b+g}{1+b}<h<b$} \\
\cline { 2 - 3 } & \multicolumn{3}{c}{ Minimum } & \multicolumn{1}{c}{ Maximum } \\
$\boldsymbol{n y y}=0.004$ & $0.063909<0.06399<0.064$ & $0.146990<0.147<0.16763$ \\
$\boldsymbol{g}=0.001$ & $0.03291<0.03295<0.033$ & $0.146992<0.147<0.17115$ \\
$\boldsymbol{g}=0.0003$ & $0.018940<0.01899<0.019$ & $0.1469991<0.147<0.17198$ \\
\hline
\end{tabular}

Now, in order to determine the bifurcations and stability conditions of the four steady states of equations (16) and (17), the non-linear equations of the model (16) - (17) are linearised to give the eigenvalues of the Jacobian's matrix,

$$
J=\left[\begin{array}{cc}
1-2 u-v-g-b v & -u(1+b) \\
-v(1-b) & 1-u-2 v+b u-h
\end{array}\right] .
$$

Firstly, the characteristic equation of the linearised system for liver-free equilibrium $(0,0)$ before therapy is given by the following quadratic equation,

$$
\begin{aligned}
\quad(1-g-\lambda)(1-h-\lambda) & =0 \\
\therefore \lambda_{1}=(1-g) & \text { or } \quad \lambda_{2}=(1-h) .
\end{aligned}
$$

For equilibrium of the above characteristic equation (18), $g>1$ and $h>1$. If $h=\frac{\delta}{r}>1$, where $r$ is the maximum proliferation rate for infected cells and $\delta$ is the infected cells death rate, then when $\delta>r$, the liver free steady state $(0,0)$ becomes a stable point implying that HCV can never totally infect the liver. However, from Table 6, it has been deduced that both $h$ and $g$ can never be greater than 1, thus the liver-free steady state $(0,0)$ can never be stable. This also leads to the idea that the total infection steady state $(0,1-h)$ is only feasible when the proliferation rate of infected cells is greater than the excess death rate of infected cells (Reluga et al., 2009) (Figure 4). 


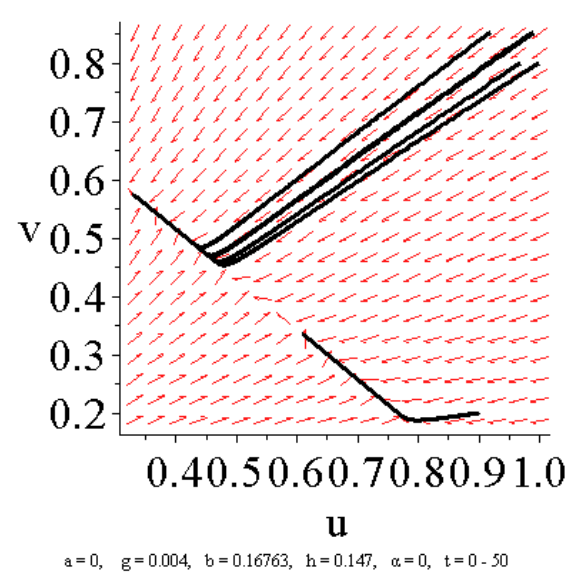

Figure 4.

Figure 4. Phase diagram corresponding to the non-dimensionalised differential equations (16) and (17) for the extended model with no migration ( $a=0)$ before therapy initiation $(\alpha=0)$. The diagram is constructed using Maple (Maplesoft, 2014) and parameter values are taken to be $g=0.004, b_{\max }=0.16763$, and $h_{\max }=0.147$ (Table 7). Since $h<1$, the total- infected steady state $(0,1-h)=(0,0.853)$ is an stable point. Here, the liver-free steady state $(0,0)$ is an unstable point.

Therefore, the first steady state that is the liver-free steady state is given by

$$
\bar{U}_{0}=(0,0) \quad \text { is }\left\{\begin{array} { c } 
{ \text { stable } } \\
{ \text { unstable } }
\end{array} \quad \text { if } \left\{\begin{array}{l}
g>1 \text { and } h>1, \\
g<1 \text { and } h<1 .
\end{array}\right.\right.
$$

Similarly, for the total- infected steady state,

$$
I_{F}=(0,1-h) \quad \text { is } \quad\left\{\begin{array} { c } 
{ \text { stable } } \\
{ \text { unstable } }
\end{array} \text { if } \left\{\begin{array}{l}
h<1, \\
h>1 .
\end{array}\right.\right.
$$

Now, we compute for the disease-free steady state, the characteristic equation of the linearised system for disease-free steady state $(1-g, 0)$ before therapy is given by the following quadratic equation,

$$
\begin{aligned}
& {[-(1-g)-\lambda][(b-h)+g(1-b)-\lambda]=0 } \\
\therefore \quad & \lambda_{1}=-(1-g) \quad \text { or } \quad \lambda_{2}=(b-h)+g(1-b) .
\end{aligned}
$$

For equilibrium of the above characteristic equation (19), $g<1$ and $(b-h)+g(1-b)<0$. Using the parameter values from Table 7, if $g=0.004, h=0.147$, and $b=0.16763$, then the condition

$(b+g)<(h+g b)$ is not satisfied such that 0.17163 is not less than 0.14767052 . Therefore, for any parameter values taken from Table 7, $(b-h)+g(1-b)$ is always greater than zero. Thus the eigenvalues for (19) are $\lambda_{1}<0<\lambda_{2}$, implying that the disease-free steady state $(1-g, 0)$ is an unstable saddle point (Figure 5). 


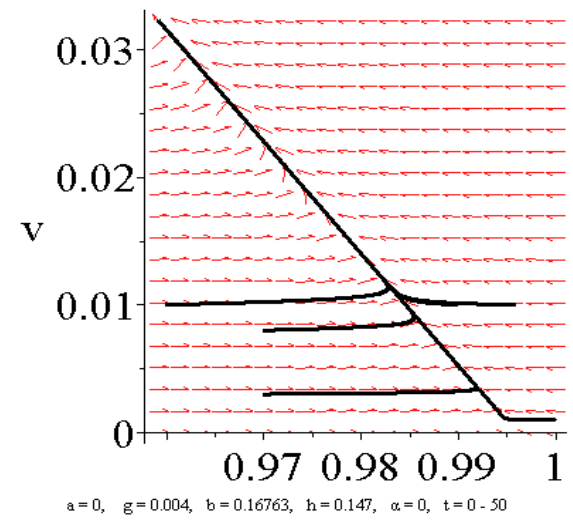

Figure 5.

Figure 5. Phase diagram corresponding to the non-dimensionalised differential equations (16) and (17) for the extended model with no migration $(a=0)$ before therapy initiation $(\alpha=0)$. The diagram is constructed using Maple (Maplesoft, 2014) and the parameter values are taken to be $g=0.004, b_{\max }=0.16763$, and $h_{\max }=0.147$ (Table 7). Since the eigenvalues of the characteristic equation (19) are $\lambda_{1}<0<\lambda_{2}$, the disease-free steady state $(1-g, 0)=(0.996,0)$ is an unstable saddle point. Here, the liver-free steady state $(0,0)$ is an unstable point Before therapy initiation $(\alpha=0)$, the partial-infected steady state $\left(\frac{h(1+b)-(b+g)}{b^{2}}, \frac{g(1-b)+(b-h)}{b^{2}}\right)$ is present when $h$ lies between $b$ and $\frac{b+g}{1+b}$. The local stability of the partial-infected steady state can be determined from the characteristic equation,

where

$$
\lambda^{2}-(A+B) \lambda+C=0,
$$

$$
C=u v(1-2 u-v-g-b v)(1-u-2 v+b u-h)\left(1-b^{2}\right) .
$$

If $0<\frac{b+g}{1+b}<h<b<1$, the constant term $(A+B)$ of (20) is negative, and the constant term of $C$ is positive.

For example, $g=0.001, h=0.147, b=0.17115$, then $u=0.00030895$ and $v=0.8527$, giving $(A+B)=-0.8529$ and $C=5.6147 \times 10^{-8}$. This implies that the characteristic equation has both negative roots (by Decartes' rule of sign) and that the partial-infected steady state is a stable nodal point (Figure 6).

During therapy $(\alpha>0)$, the steady states are the liver-free steady state $(0,0)$, disease-free solution $(1-g, 0)$, total infection solution $(0,1-\mathrm{h})$ and partial-infection solution $\left(\frac{h[1+b(1-\alpha)]-(b(1-\alpha)+g)}{(1-\alpha)^{2} b^{2}}, \frac{g[1-b(1-\alpha)]+(b(1-\alpha)-g)}{(1-\alpha)^{2} b^{2}}\right)$.

The phase portrait (Figure 7) below illustrates the stability of the steady states.

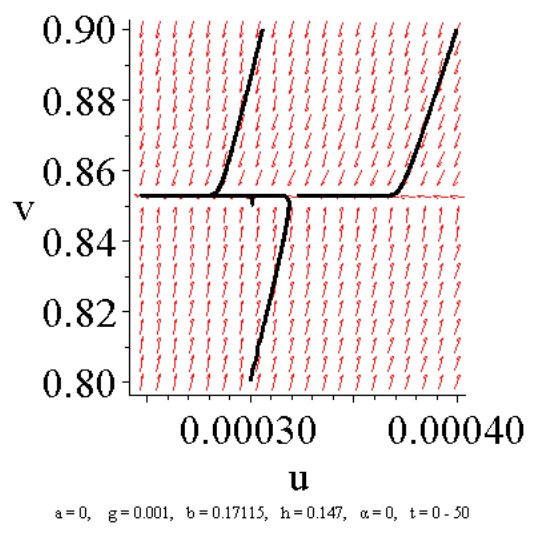

Figure 6. 
Figure 6. Phase diagram corresponding to the non-dimensionalised differential equations (16) and (17) for the extended model with no migration $(\mathrm{a}=0)$ before therapy initiation $(\alpha=0)$. The diagram is constructed using Maple (Maplesoft, 2014) and the parameter values are taken to be $g=0.001$, bmax $=0.17115$, and hmax $=$

0.147 (Table 7). While, the partial infected steady state $\left(\frac{h(1+b)-(b+g)}{b^{2}}, \frac{g(1-b)+(b-h)}{b^{2}}\right)=(0.00030895$, 0.85274 ) is a stable nodal point.

For $100 \%$ drug efficacy $(\alpha=1.0)$, the steady states are the liver-free steady state $(0,0)$, disease-free solution $(1-g, 0)$, total infection solution $(0,1-h)$. The phase portrait (Figure 8$)$ below illustrates the stability of the steady states.

Before therapy $(\alpha=0)$ and with migration $(a>0)$, the steady states are $\left(\frac{1-h}{1-b}, 0\right), \quad\left(\frac{(1-g)+\sqrt{(g-1)^{2}+4 a}}{2}, 0\right)$, $\left(\frac{(1-g)-\sqrt{(g-1)^{2}+4 a}}{2}, 0\right)$, and the other two steady states are $u=\frac{[(1-g)-(1-h)(1+b)] \pm \sqrt{[(1-g)-(1-h)(1+b)]^{2}+4 a b^{2}}}{2 b^{2}}$ with $v=(1-h)-(1-b) u$. The phase portrait (Figure 9) below illustrates the stability of the steady states.

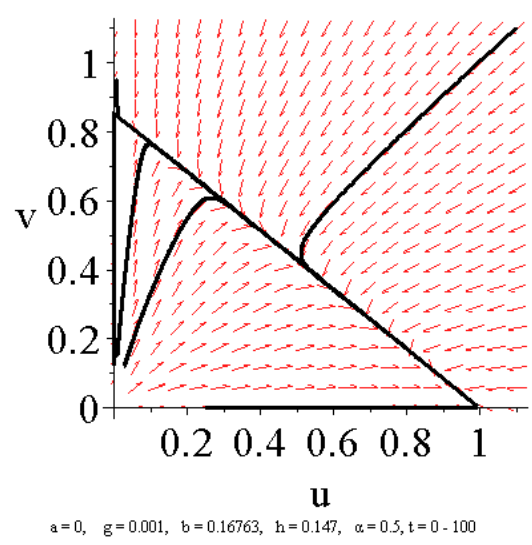

Figure 7.

Figure 7. Phase diagram corresponding to the non-dimensionalised differential equations (16) and (17) for the extended model with no migration $(\mathrm{a}=0)$ and $50 \%$ drug efficacy $(\alpha=0.5)$. The diagram is constructed using Maple (Maplesoft, 2014) and the parameter values are taken to be $g=0.001$, and hmax $=0.147$ (Table 7). Since $\mathrm{h}<1$, the total infection steady state $(0,1-\mathrm{h})$ is a stable point so HCV can possibly fully infect the liver. Similarly, the liver-free steady state $(0,0)$ is again an unstable steady state. With $50 \%$ drug efficacy, there is significant treatment.

Table 8. Stationary solutions for Dahari et al. (2007) model and their characteristics

\begin{tabular}{lcc}
\hline Stationary points & Location & $\begin{array}{l}\text { Local stability condition } \\
\text { (stable only) }\end{array}$ \\
\hline $\begin{array}{l}\text { Liver-free solution } \\
\text { Disease-free solution }\end{array}$ & $(0,0)$ & Never stable \\
Total-infection solution & $(1-g, 0)$ & $g=\frac{d}{r}<1$ \\
Partial-infection solution & $\left(\frac{h(1+b)-(b+g)}{b^{2}}, \frac{g(1-b)+(b-h)}{b^{2}}\right)$ & $h=\frac{\delta}{r}<1$ \\
& & $0<\frac{b+g}{1+b}<h<b<1$ \\
\hline
\end{tabular}




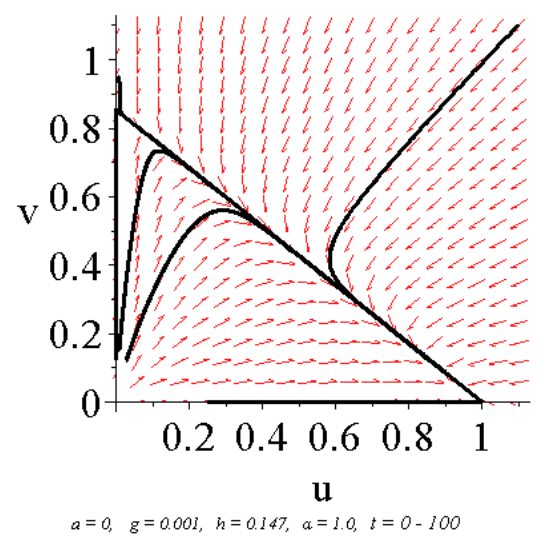

Figure 8 .

Figure 8. Phase diagram corresponding to the non-dimensionalised differential equations (16) and (17) for the extended model with no migration $(a=0)$ and $100 \%$ drug efficacy $(\alpha=1)$. The diagram is constructed using Maple (Maplesoft, 2014) and the parameter values are taken to be $g=0.001$, and hmax $=0.147$ (Table 7). Here, the liver-free steady state $(0,0)$ is also an unstable steady state. With $100 \%$ drug efficacy, the only stable steady state is the disease-free equilibrium.

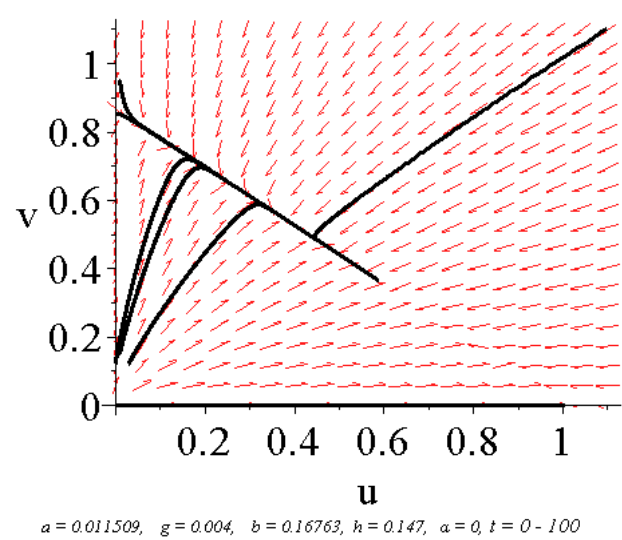

Figure 9.

Figure 9. Phase diagram corresponding to the non-dimensionalised differential equations (16) and (17) for the extended model with migration $(\mathrm{a} \neq 0)$ before therapy $(\alpha=0)$. The diagram is constructed using Maple (Maplesoft, 2014) and the parameter values are taken to be $g=0.004$, and hmax $=0.147$ (Table 8 ). The steady states are $(1.025,0),(1.007,0),(-0.0114,0),(0.6402,0.320)$ and $(-0.6398,1.036)$.

\section{Discussion}

The diversity of the disease manifestation associated in the mathematical models of HCV strongly suggested that, the infection is determined by the ranges of values of parameters that govern the infected patient response to therapy. A detailed analysis on the local stability of the mathematical models has been carried out.

In the original model proposed by Neumann et al. (1998), it is shown that, if $\mathrm{R} 0<1$, the disease-free steady state before therapy is asymptotically stable. If R $0>1$, then the chronically infected steady state before therapy is asymptotically stable. Therefore, we have a threshold phenomenon, that is, if $\mathrm{R} 0<1$, the infected steady state is not feasible while if R0 $>1$, infection ensues. The basic production rate of the infection, R0 is a crucial parameter in this model in dealing with the stability of the spread of the HCV infection. Furthermore, during therapy, the partial-infected steady state can become unstable (viral load approaches zero) if $0<(1-\varepsilon)(1-$ $\eta) R_{0}<1$. An important implication of this analysis is that, the infection can only be controlled with the constant presence of the antiviral therapy. Once the treatment ceases, the virus may return to the pre-treatment level which, indicate that viral eradication is highly not possible in this model. Similarly, the assumption made by Neumann 
et al. (1998) that the target cells remain constant during therapy does not account for the liver, as an organ that compensate for the loss of infected cells, by proliferation of hepatocytes. Therefore, the original model needs to incorporate some general aspects of immunology control, effect of resistance to infection, and vaccination strategies (Murray, 2002).

A more realistic model has been proposed by Dahari et al. (2007) as the extended model, to account for the maximum proliferation rate of hepatocytes ( $\mathrm{r}$ ). In this model, when the disease-free reproductive number $g=\frac{d}{r}$ is less than 1 , then infection is not feasible, because the maximum proliferation rate of uninfected cells are greater than the death rate of the uninfected cells. However, when an individual become fully infected (total infection), virus will infect the entire target cells, and remain infected (stable) if $\mathrm{h}=\frac{\delta}{\mathrm{r}}<1$ (maximum proliferation rate of infected cells are greater than death rate of infected cells). According to Dahari et al. (2005), when an infected individual is treated with IFN in early initiation ( 89 days), there is $100 \%$ drug efficacy. This is shown in Figure 6 where disease-free steady state is the only stable equilibrium, when drug efficacy is $100 \%$ and treated within 100 days. Here, we have analysed a model with experimentally estimated parameters that, provide a satisfactory fit of the steady states corresponding to biological meanings, and successful feasibility testing of this model.

It is clear that mathematical modelling of viral response to antiviral therapy can be used to understand the dynamics of chronic viral infection (Layden, Mika \& Wiley, 2000). The approach to study the stability of the mathematical model is the reasonable starting point, for understanding the dynamics of HCV in pertaining a vaccine or cure for the disease. A combined analysis of HCV dynamics and viral kinetic studies, may prove useful in further work of understanding sustained viral response (SVR), and shed important light on the scale of patients responding to therapy. This may be the critical analysis for estimating the drug efficacy within the critical value that ultimately should eradicate the HCV infection.

\section{Acknowledgements}

Part of this work was carried out as part of Maureen Chong Master's study at University of Sussex. MC acknowledges the support and scholarship grant from The Government of His Majesty the Sultan and Yang Di-Pertuan Negara Brunei Darussalam. Anotida Madzvamuse acknowledges support from the Engineering and Physical Sciences Research Council Grant (EP/J016780/1) and the Leverhulme Trust Research Project Grant (RPG-2014-149). Laurie Crossley is grateful for support from the School of Mathematical and Physical Sciences and Life Sciences at the University of Sussex.

\section{References}

Avendaño, R., Esteva, L., Flores J. A., Fuentes Allen, J. L., Gómez, G., \& López-Estrada, J. A. (2002). Mathematical model for the dynamic of hepatitis C. Journal of Theoretical Medicine, 4, 109-118. http://dx.doi.org/10.1080/10273660290003777

Bisceglie, D. A. M. (1998). Hepatitis C. The Lancet, 351, 351-355. http://dx.doi.org/10.1016/S0140-6736(97)07361-3

Brauer, F. (2004). The analysis of some characteristic equations rising in population and epidemic models. Journal of Dynamics and Differential Equations, 16, 441-453. http://dx.doi.org/10.1007/s10884-004-4287-z

Centers for Disease Control and Prevention (CDC), USA. (2009). Hepatitis C - FAQs for Health Professionals. Retrieved from http://www.cdc.gov/ hepatitis/HCV/HCVfaq.htm\#section1

Chatterjee, A., Guedj, J., \& Perelson, A. S. (2012). Mathematical modelling of HCV infection: What can it teach us in the era of direct-acting antiviral agents? Antiviral Therapy, 17(6PtB), 1171-1182. http://dx.doi.org/10.3851/IMP2428

Cole, W. H., \& Everson, T. C. (1966). Spontaneous Regression of Cancer. WB Saunders, Philadelphia.

Dahari, H., Layden-Almer, J. E., Kallwitz, E., Ribeiro, R. M., Cotler, S. J., Layden, T. J., \& Perelson, A. S. (2009). A mathematical model of hepatitis $C$ virus dynamics in patients with high baseline viral loads or advanced liver disease. Gastroenterology, 136, 1402-1409. http://dx.doi.org/10.1053/j.gastro.2008.12.060

Dahari, H., Lo, A., Ribeiro, R. M., \& Perelson, A. S. (2007). Modeling hepatitis C virus dynamics: Liver regeneration and critical drug efficacy. Journal of Theoretical Biology, 247, 371-381. http://dx.doi.org/10.1016/j.jtbi.2007.03.006

Dahari, H., Major, M., Zhang, X., Mihalik, K., Rice, C. M., Perelson, A. S., Feinstone, S. M., \& Neumann, A. U. (2005). Mathematical modeling of primary hepatitis C infection: Noncytolytic clearance and early blockage 
of virion production. Gastroenterology, 128, 1056-1066. http://dx.doi.org/10.1053/j.gastro.2005.01.049

Firpi, R. J., \& Nelson, D. R. (2007). Current and future hepatitis C therapies. Archives of Medical Research, 38, 678-690. http://dx.doi.org/10.1016/j.arcmed.2006.09.002

Guedj, J., \& Neumann, A. U. (2010). Understanding hepatitis C viral dynamics with direct-acting antiviral agents due to the interplay between intracellular replication and cellular infection dynamics. Journal of Theoretical Biology, 267(3), 330-340. http://dx.doi.org/10.1016/j.jtbi.2010.08.036

Guedj, J., Dahari, H., Rong, L., Sansone, N. D., Nettles, R. E., Cotler, S. J., Layden, T. J., Uprichard, S. L, \& Perelson, A. S. (2013). Modelling shows that the NS5A inhibitor daclatasvir has two modes of action and yields a shorter estimate of the hepatitis C virus half-life. Proceedings of the National Academy of Sciences of the United States of America, 110(10), 3991-3996. http://dx.doi.org/10.1073/pnas.1203110110

Guedj, J., Rong, L., Dahari, H., \& Perelson, A. S. (2010). A perspective on modelling hepatitis C virus infection. Journal of Viral Hepatitis, 17, 825-833. http://dx.doi.org/10.1111/j.1365-2893.2010.01348.x

Hale, J., \& Kocak, H. (1991). Dynamics and Bifurcations, Springer-Verlag. http://dx.doi.org/10.1007/978-1-4612-4426-4

Kot, M. (2001). Elements of Mathematical Ecology. Cambridge University Press. http://dx.doi.org/10.1017/CBO9780511608520

Layden, T. J., Mika, B., \& Wiley, T. (2000). Hepatitis C kinetics: Mathematical modeling of viral response to therapy. Seminars in Liver Disease, 20(2), 173-183. http://dx.doi.org/10.1055/s-2000-9940

Maplesoft. (2014). Retrieved from www.maplesoft.com/index.aspx.

Mihm, U., Hermann, E., Sarrazin, C., \& Zeuzem, S. (2006). Review article: predicting response in hepatitis C virus therapy. Alimentary Pharmacology \& Therapeutics, 23(8), 1043-1054. http://dx.doi.org/10.1111/j.1365-2036.2006.02863.x

Murray, J. D. (2002). Mathematical Biology I: An Introduction (3rd ed). Springer.

Neumann, A. U., Lam, N. P., Dahari, H., Gretch, D. R., Wiley, T. E., Layden, T. J., \& Perelson, A. S. (1998). Hepatitis C viral dynamics in vivo and the antiviral efficacy of interferon- $\alpha$ therapy. Science, 282, 103-107. http://dx.doi.org/10.1126/science.282.5386.103

Reluga, T. C., Dahari, H., \& Perelson, A. S. (2009). Analysis of hepatitis C virus infection models with hepatocyte homeostasis. SIAM Journal on Applied Mathematics, 69(4), 999-1023. http://dx.doi.org/10.1137/080714579

Rong, L., \& Perelson, A. S. (2013). Mathematical analysis of multiscale models for hepatitis C virus dynamics under therapy with direct-acting antiviral agents. Mathematical Biosciences, 245(1), 22-30. http://dx.doi.org/10.1016/j.mbs.2013.04.012

Soriano, V., Perelson, A. S., \& Zoulim, F. (2008). Why are there different dynamics in the selection of drug resistance in HIV and hepatitis B and C viruses? Journal of Antimicrobial Chemotherapy, 62, 1-4. http://dx.doi.org/10.1093/jac/dkn175

Strader, D. B., Wright, T., Thomas, D. L., \& Seeff, L. B. (2004). Diagonis, management, and treatment of hepatitis C, Hepatology, 39(4), 1147-1171. http://dx.doi.org/10.1002/hep.20119

World Health Organization (WHO). (1999). Hepatitis C - global prevalence (update), The Weekly Epidemiology Record, 74(49), 425-427.

World Health Organization (WHO). (2014). Hepatitis C, Fact Sheet No. 164. Retrieved from http://www.who.int/mediacentre/factsheets/fs164/en/

\section{Copyrights}

Copyright for this article is retained by the author(s), with first publication rights granted to the journal.

This is an open-access article distributed under the terms and conditions of the Creative Commons Attribution license (http://creativecommons.org/licenses/by/3.0/). 